\section{Sahara, Western}

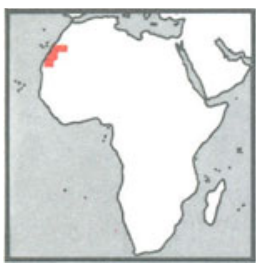

Location North-west Africa

With a coastline on the Atlantic Ocean,

Morocco is to the north, Algeria has a small

common border to the north-east, and

Mauritania is to the east and south

Land Area $266000 \mathrm{~km}^{2}=103000 \mathrm{mi}^{2}$

Climiate Hot and dry

Weather at Dakhlah, 11 m altitude

Temperature: hottest month September

$19-27^{\circ} \mathrm{C}$, coldest January $13-22^{\circ} \mathrm{C}$

Rainfall (av monthly): driest month June $0 \mathrm{~mm}$, wettest September $36 \mathrm{~mm}$ Time GMT

Measures Metric system

Monetary unit Moroccan dirham and Mauritanian ouguiya

Summary

Political Territory administered by Morocco and Mauritania under an agreement made November 14, 1975 with Spain; formerly a Spanish province, Spain handed over full administration on February 26, 1976. There is a guerrilla movement fighting for independence as the Sahraoui Republic

Economic Phosphates are the most important feature, production having begun in 1972 ; the mainly nomadic population subsists by raising livestock. Guerrilla activity reduced the production of phosphates from 1976

\section{People, resources and equipment}

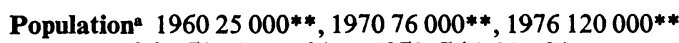

Growth: 1960-70 11.8**\%pa, 1970-76 8.0**\%pa

Density $\left((1976): 0.5^{* *}\right.$ people per $\mathrm{km}$ *

Vital statistics (rate per 1000 people, 1972): births $20.9^{*}$, deaths $4.5^{*}$

Estimates are very approximate due to the number of nomads in the population; excludes refugees outside of the territory

Cities (population in 000,1974) El Aaiún 28, Smara 7*, Dakhlah" 5*

- Called Villa Cisneros before January 12, 1976

Race (1970) Arab $78 \%$ European $22 \%$

Language (1970) Arabic and Hassania $78 \%$, Spanish $22 \%$

Religion (1970) Moslem $78 \%$, Roman Catholic $22 \%$

Education (1972/73) Pupils 5 270, teachers 260*

Personnel (1971) Physicians: 53, 1 per 1570 people

National income per person (1976) $\mathrm{Dh} 4000^{* * *}=\$ 900^{* * *}=£ 500^{* * *}$

Consumption per person (1975) Electricity $570^{*} \mathrm{~kW} \mathrm{~h}$

Livestock $(000,1976)$ Goats $152^{*}$, sheep 17*, camels 83*

Mineral reserves (1974) Phosphates $1700^{* *} \mathrm{mn}$ tonnes

Electrical capacity (1975) $52 *$ megawatts

Hospital beds (1972) 262, 1 per 344 people

Roads (1974) $6500 * \mathrm{~km}=4000^{*} \mathrm{mi}$, density $0.02 * \mathrm{~km}$ per km ${ }^{2}$

There are no railways

Port Dakhlah

Airports El Aaiún, Dakhlah

Durable equipment 000 no per

(Dec 1974)

Radio sets

Television sets

1000 people

Passenger cars

$16 \quad 144$

44 people no per

0.8

\section{Production, finance and trade}

Gross domestic product

1976 est: Dh $500^{* * *} \mathrm{mn}=\$ 110^{* * *} \mathrm{mn}=£ 60 * * * \mathrm{mn}$

Main products $(000 \mathrm{t}, 1975)$ Barley $1^{*}$, goat milk $41^{*}$, fish catch $4^{*}$,

electricity $(\mathrm{mn} \mathrm{kW} \mathrm{h}) 65^{*}$, phosphates $3300^{*}$

Transport traffic (1974) Sea Goods loaded $624000 \mathrm{t}$,

unloaded $387000 \mathrm{t}$

Budget (1973) Balanced at $\mathrm{Pa} 1553 \mathrm{mn}=\$ 26 \mathrm{mn}=£ 11 \mathrm{mn}$

External trade (1976)

Imports: $\mathrm{Dh} 450^{* * *} \mathrm{mn}=\$ 100^{* * *} \mathrm{mn}=£ 60^{* * *} \mathrm{mn}$

Exports: $\mathrm{Dh} 450 * * * \mathrm{mn}=\$ 100^{* * *} \mathrm{mn}=£ 60^{* * *} \mathrm{mn}$

Main imports (\% of total, 1973) Food 54, manufactures 44

Main export: Phosphates

Saint Helena

Colony of Saint Helena and dependencies

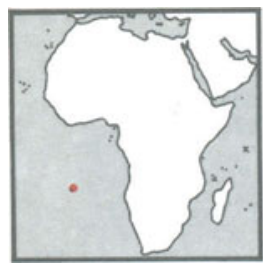

Location South Atlantic

The island of St Helena is $2000 \mathrm{~km}$ west of Angola with Ascension island $1100 \mathrm{~km}$ to the north-west and the island of

Tristan da Cunha $2400 \mathrm{~km}$ to the south-west

Land Area $413 \mathrm{~km}^{2}=160 \mathrm{mi}^{2}$

of which, St Helena $122 \mathrm{~km}^{2}$, Ascension $88 \mathrm{~km}^{2}$,

Tristan da Cunha $98 \mathrm{~km}^{2}$

Climate Mild, temperate in Tristan da Cunha

Weather at Jamestown, 12 m altitude

Temperature: hottest month March $22-28^{\circ} \mathrm{C}$, coldest July-Sept $17-22^{\circ} \mathrm{C}$ Rainfall (av monthly): driest month Nov $0 \mathrm{~mm}$, wettest March $20 \mathrm{~mm}$ Time GMT

Measures UK (imperial) system, changing to the metric system in line with the United Kingdom

Monetary unit UK pound $(£)=100$ new pence

Rate of exchange (1976 av): free $\$ 1.806=£ 1$

\title{
Summary
}

Political St Helena is a UK colony, and Ascension, Tristan da Cunha, Gough, Inaccessible and Nightingale islands are dependencies. Ascension Island is also sometimes known as Wideawake Island. Tristan da Cunha was evacuated from 1961-63 due to a volcanic eruption

Economic There is no industry nor are there minerals; agricultural production is very limited. There are telecommunications and meteorological stations, and a US air and missile base

\section{People, resources and equipment}

Population $19605300 * *, 19706100 * *, 19766600^{* *}$

Growth: 1960-70 1.4**\%pa, 1970-76 1.3**\%pa

Density (1976): $16^{* *}$ people per km

Vital statistics (rate per 1000 people, St Helena only, 1975)

births 25.0 , deaths 8.1

Regions (population in 000, 1975) St Helena 5.1, Ascension 1.2*,

Tristan da Cunha $0.3^{*}$; Gough, Inaccessible and Nightingale islands : uninhabited

Town (population in 000, 1976) Jamestown (capital) $1.6^{*}$

Race (1976) St Helenians 85*\%

Language English

Religion (1966) Christian $99.6 \%$ (mainly Anglican Communion)

Education (1974) Pupils $1250^{*}$, teachers $71^{*}$

Labour force (employees, 1975) 1667

Personnel (1973) Physicians: 3, 1 per 2160 people

National income per person (1976) $£ 300 * * *=\$ 550 * * *$

Consumption per person (1975) Electricity $150 * \mathrm{~kW} \mathrm{~h}$

Livestock (000, Dec 1975) Cattle 1, sheep 3, goats 2, poultry 11

Electrical capacity (1972) 0.45 megawatt

Hospital beds (1975) 54, 1 per 121 people

Roads (1975) $107 \mathrm{~km}=67 \mathrm{mi}$, density $0.26 \mathrm{~km}$ per $\mathrm{km}$ *

Port Jamestown

Airport There is an airfield on Ascension

Durable equipment (Dec 1974) Radio sets: 750,115 per 1000 people

Motor vehicles: 791,121 per 1000 people, 7.4 per km of road

Production, finance and trade

Gross domestic product 1976 est : $£ 2.0^{* * *} \mathrm{mn}=\$ 3.6^{* * *} \mathrm{mn}$

Main products (1975) Potatoes, sweet potatoes, vegetables, fish $162 \mathrm{t}$, electricity $1 * \mathrm{mn} \mathrm{k} \mathrm{W} \mathrm{h}$

Transport traffic Sea (1974) Goods unloaded $11000 \mathrm{t}$

Budget (1975/76: year ending March 31st)

Revenue: $£ 1482000=\$ 3148000$

Expenditure: $£ 1544000=\$ 3279000$

External trade (1975) Imports: $£ 1.1 \mathrm{mn}=\$ 2.5 \mathrm{mn}$. Exports : nil

Main imports (1974)

Manufactures

(of which, motor vehicles 6)

Food

$\%$ of total Main exports (1974)

16

Beer, wines and spirits

Main sources (1968)

United Kingdom

South Africa

United States

$45 *$

0*

7

28
None (in 1968 there

were exports of

wool and fish)

Main destinations (1974)

None (in 1968: United

Kingdom $78 \%$

South Africa $22 \%$ 